\title{
Serum creatinine level, a surrogate of muscle mass, predicts mortality in critically ill patients
}

\author{
Charat Thongprayoon ${ }^{1}$, Wisit Cheungpasitporn ${ }^{1}$, Kianoush Kashani ${ }^{1,2}$ \\ ${ }^{1}$ Division of Nephrology and Hypertension, ${ }^{2}$ Division of Pulmonary and Critical Care Medicine, Department of Internal Medicine, Mayo Clinic, \\ Rochester, MN, USA \\ Correspondence to: Kianoush Kashani, MD. Mayo Clinic, 200 First Street SW, Rochester, MN 55905, USA. Email: kashani.kianoush@mayo.edu.
}

\begin{abstract}
Serum creatinine (SCr) has been widely used to estimate glomerular filtration rate (GFR). Creatinine generation could be reduced in the setting of low skeletal muscle mass. Thus, SCr has also been used as a surrogate of muscle mass. Low muscle mass is associated with reduced survival in hospitalized patients, especially in the intensive care unit (ICU) settings. Recently, studies have demonstrated high mortality in ICU patients with low admission SCr levels, reflecting that low muscle mass or malnutrition, are associated with increased mortality. However, SCr levels can also be influenced by multiple GFR- and nonGFR-related factors including age, diet, exercise, stress, pregnancy, and kidney disease. Imaging techniques, such as computed tomography (CT) and ultrasound, have recently been studied for muscle mass assessment and demonstrated promising data. This article aims to present the perspectives of the uses of SCr and other methods for prediction of muscle mass and outcomes of ICU patients.
\end{abstract}

Keywords: Creatinine; cystatin; intensive care unit (ICU); malnutrition; mortality; muscle mass

Submitted Feb 12, 2016. Accepted for publication Mar 02, 2016.

doi: $10.21037 /$ jtd.2016.03.62

View this article at: http://dx.doi.org/10.21037/jtd.2016.03.62

\section{Introduction}

Creatinine is an endogenous substance generated from the nonenzymatic conversion of creatine and creatine phosphate, $95 \%$ of which is found in muscle (1). Creatinine is an uncharged, small molecular weight substance (113 Da) that is not bound to serum proteins. It is filtered freely by the glomerulus without tubular reabsorption. Creatinine is also secreted by the renal tubules only in small amounts (1).

The serum creatinine (SCr) level is ubiquitously used to estimate glomerular filtration rate (GFR) during a steady state of renal function. Although elevated SCr could be due to changes in its secretion (impact of medications: probenecid, cimetidine, trimethoprim) or production (due to increased muscle mass or meat consumption), its rise usually indicates either acute kidney injury (AKI) or chronic kidney disease. SCr-based AKI definitions have demonstrated consistent power in predicting mortality and other outcomes among the hospitalized patients (2-8). Due to the correlation between SCr levels and muscle mass, SCr in the steady state has been used as a surrogate of muscle mass measurements (9). Creatinine generation is low among individuals who have more diminutive muscle mass, either constitutionally or disease-related. Therefore, low SCr level could be considered as a proxy of protein-energy wasting in some clinical situations (10). This article discusses the perspectives of the utility of SCr and other methods as predictors of muscle mass and outcomes of intensive care unit (ICU) patients.

\section{Mechanism of low serum creatinine (SCr) level}

Low SCr levels are associated with multiple factors as shown in Table 1 (10-15). As previously stated, creatinine generation could be reduced in the setting of low muscle mass. In the other words, malnourished individuals with smaller muscle mass have lower SCr levels. Muscle mass is related to gender (females may have less muscle mass), age (advancing age may be associated with decreasing muscle mass), and ethnic background (African Americans tend to 
Table 1 Factors associated with low serum creatinine levels

Low muscle mass (female gender, elderly, chronic illness)

Malnutrition

Vegetarian diet

Pregnancy

Advanced liver disease

Fluid overload

Augmented renal clearance

have higher muscle mass) (10). Individuals' SCr levels can also be affected by diet. Arginine and glycine are creatine precursors. Therefore, low dietary protein intake can limit creatinine generation. Also, cooked meat contains a significant amount of creatinine, which is absorbed in the intestinal tract. Thus, protein malnutrition could result in low SCr levels (11). A high GFR, as in pregnancy, could also lower SCr levels (10-12).

Patients with advanced liver diseases can have low $\mathrm{SCr}$ due to diminished creatinine production from decreased hepatic creatine synthesis, enhanced tubular creatinine secretion, and reduced skeletal muscle mass (12). Creatinine is distributed in total body water, and large fluid volume resuscitation, as is often required in the sickest ICU patients, could increase the volume of distribution of creatinine, resulting in lowered SCr values (13). Chronic illness, age, malnutrition, and pathologic conditions such as protein-losing disorders like enteropathies and nephrotic syndrome, also impact muscle mass and creatinine production (11).

Augmented renal clearance (ARC), an enhanced elimination of solutes by the kidneys at a rate significantly higher than normal, is a phenomenon whereby patients experience marked increase in functional creatinine clearance and GFR in acute illness; thus, leading to low SCr levels (14). With reported incidence rates ranging from $16 \%$ to $100 \%$, ARC is commonly observed among ICU patients (15). Systemic inflammatory response syndrome (SIRS) is a common cause of ARC in critically ill patients (14).

\section{Low serum creatinine (SCr) levels and mortality}

Cartin-Ceba et al. (16) have previously reported the results of a large retrospective cohort study of 11,291 patients admitted to Mayo Clinic Hospital-Rochester ICUs between 2003 and 2006, evaluating the association between baseline SCr concentration at admission to ICU and inhospital mortality. Both low and high baseline SCr levels were associated with increased in-hospital mortality. Multivariable regression analysis was used to adjust for various relevant variables including body mass index (BMI). The noted low baseline SCr was independently associated with increased mortality in a dose-response fashion. The investigators postulated that the association was due to diminished muscle mass and malnutrition.

Recently, Udy et al. (17) reported a large retrospective study of $1,045,718$ patients across 172 ICUs by exploring data from the prospective Australian and New Zealand Intensive Care Society Centre for Outcome and Resource Evaluation adult patient database. To mitigate the impact of volume resuscitation on SCr levels during ICU admission, the investigators stratified patients based on the peak recorded SCr concentration during the first $24 \mathrm{~h}$ of ICU admission, rather than the lowest SCr levels. Using a reference $\mathrm{SCr}$ value of $0.79-0.89 \mathrm{mg} / \mathrm{dL}$, the investigators reported a progressively increased risk of ICU mortality at peak admission SCr levels $<0.68 \mathrm{mg} / \mathrm{dL}$ and $\mathrm{SCr}$ levels $<0.34 \mathrm{mg} / \mathrm{dL}$ [odds ratio (OR) for in-hospital mortality $=2.03 ; 95 \%$ CI, 1.86-2.21). The study of SCr levels as a predictor of ICU or hospital outcomes is associated with limitations, as mentioned earlier. Fluid resuscitation is very prevalent in the ICU, especially within $24 \mathrm{~h}$ of ICU admission, so using peak SCr levels cannot completely eliminate this potential confounder. Also, low SCr levels may represent ARC; this would potentially interfere with maintaining therapeutic antimicrobial concentrations, which could be potentially associated with increased ICU mortality (14). Despite these limitations, it could be reasonably postulated that low SCr level on ICU admission reflects low muscle mass or malnutrition, which are associated with increased mortality.

Previous studies have demonstrated that high SCr levels in hemodialysis patients are associated with greater survival, whereas low SCr levels are associated with increased mortality $(18,19)$. In the Alberta Kidney Disease Network (AKDN) study among >900,000 Canadians, Tonelli et al. (20) found low SCr levels with eGFR $\geq 105 \mathrm{~mL} / \mathrm{min} / 1.73 \mathrm{~m}^{2}$ were associated with increased mortality. Also, low SCr levels have been correlated with cardiovascular diseases $(21,22)$. Recently, Choi et al. (23) conducted a cross-sectional study of 6,986 middle-aged Korean men, which showed a U-shaped association between eGFR and advanced coronary artery calcification, as measured by computed tomography (CT). Compared with study individuals who 
had eGFRs between 75 and $89 \mathrm{~mL} / \mathrm{min} / 1.73 \mathrm{~m}^{2}$, those with either lower or higher eGFRs were at increased risk for coronary artery calcium scores above 100. Even after adjustment for confounders, individuals with low SCr levels and eGFRs $\geq 105 \mathrm{~mL} / \mathrm{min} / 1.73 \mathrm{~m}^{2}$ had an OR of 2.53 for advanced coronary artery calcification, compared with subjects with eGFRs between 75 and $89 \mathrm{~mL} / \mathrm{min} / 1.73 \mathrm{~m}^{2}$. However, the data on proteinuria, an important marker of kidney damage, was not available in this study (23). Proteinuria, particularly albuminuria, has been shown to be associated with higher mortality and acute myocardial infarction (20). In addition, a SCr-based GFR equation is affected by non-GFR determinants of SCr including diet, muscle metabolism, and metabolic disorders. Combined analysis revealed that higher mortality in non-critically ill patients with low SCr levels likely results from malnutrition and illness, not from enhanced kidney function. Although future studies are required to assess the impact of changes in the GFR of the outcomes, in studies used cystatin C (CysC), which is independent of muscle metabolism and diet, there is a linear, not a U-shaped, association between eGFR and adverse events (24).

\section{Muscle mass, nutritional status, and mortality}

Skeletal muscle, accounting for $40 \%$ of body weight and $50 \%$ of body protein, plays a vital role in regulating immune function, glucose disposal, protein synthesis and mobility (25). Muscle provides a massive dynamic reservoir of proteins, minerals, and other intermediate metabolites that can be cannibalized to meet the need of other tissues involved in the inflammatory response. Loss of skeletal muscle and the reduced protein reservoir may predispose impaired tissue healing and poor immune function (26). As more than $75 \%$ of glucose metabolism is handled by skeletal muscle, its atrophy can impair insulin signaling, and glucose tolerance (27).

Studies have shown the reduced survival rates and the increased hospital lengths of stay of patients who have a poor nutrition status and low muscle mass $(26,28,29)$. In patients with low muscle mass and malnutrition, cardiovascular outcomes are generally poor (30), life expectancy in cancer is reduced (31), and outcomes following liver transplantation are unfavorable (32). In elderly patients, sarcopenia, the age-associated loss of skeletal muscle mass and function, is associated with higher morbidities and mortalities (33).

Malnutrition and wasted muscles are common features in ICU patients, due to a protracted catabolic condition, correlated with high morbidity and mortality. The critical illness-related hypercatabolic state does not improve by just providing adequate nutritional support (34). Inflammatory cytokines in the setting of SIRS/sepsis have an established role in regulating muscle mass. TNF- $\alpha$, IL-1, IL- 6 , and endotoxin infusions result in muscle wasting syndrome due to increased protein catabolism, inhibition of protein synthesis, inhibition of muscle cell differentiation, or reduced amino acid uptake (35). ICU-related respiratory muscle wasting leads to difficulties in weaning patients from mechanical ventilation (36).

\section{Assessment of muscle mass and nutritional status in ICU}

Critically ill patients require special considerations during muscle mass assessment. Table 2 shows the typical methods used for nutritional status and muscle mass assessment in ICUs (37-48). Several studies have indicated the tools typically used to assess nutritional status are poor indicators of malnutrition in the critically ill population $(37,49-51)$. Skeletal muscle wasting in the ICU is frequently masked by excess fat (sarcopenic obesity) (52), or by fluid retention that can amount to $10-20 \%$ of the patient's body weight (53). As discussed earlier, SCr levels are influenced by age, diet, exercise, stress, and renal disease and require cautious interpretations. Having low BMI and weight are also identified risk factors for death in ICU patients (38). Unfortunately, many ICU patients are edematous, and the measured weight and BMI may not reflect the real body muscle mass (39). Interpretation of results of other anthropometric measurements such as mid-upper arm circumference and triceps skinfold thickness also remains uncertain and of limited value to the ICU setting, as the techniques all assume a normal state of hydration (54). In addition, since ICU patients are frequently sedated, voluntary muscle strength tests cannot be performed because of impaired patient cooperation.

Albumin is also a poor marker of nutritional status, especially in the ICU setting, due to changes in intravascular volume, as well as other factors, including the impact of acute infection, inflammation, hepatic function, and proteinlosing states (40). The use of tools that assess muscle mass and nutrition, such as subjective global assessment (SGA) and Nutrition Risk in Critically Ill Score (NUTRIC) (37) has been proposed. However, screening and evaluation tools often have components that are difficult to obtain in 
Table 2 Assessment of muscle mass and nutritional status in intensive care unit (ICU)

\begin{tabular}{|c|c|c|}
\hline Characteristics & $\begin{array}{l}\text { Tools for muscle mass } \\
\text { assessment in ICU }\end{array}$ & $\begin{array}{l}\text { Tools for nutritional } \\
\text { status in ICU }\end{array}$ \\
\hline Body mass index (BMI) & $\mathrm{X}$ & $\mathrm{X}$ \\
\hline Muscle biopsies & $\mathrm{X}$ & \\
\hline Muscle strength tests & & $\mathrm{X}$ \\
\hline Nutrition Risk in Critically III Score (NUTRIC) & & $x$ \\
\hline Nutrition-focused physical assessment (NFPA) & & $\mathrm{X}$ \\
\hline $\begin{array}{l}\text { Physical assessment and anthropometric measurements (mid-upper } \\
\text { arm circumference and triceps skinfold thickness) }\end{array}$ & $\mathrm{X}$ & $\mathrm{X}$ \\
\hline Serum alanine aminotransferase (ALT) & $x$ & \\
\hline Subjective global assessment (SGA) & & $\mathrm{X}$ \\
\hline
\end{tabular}

$\mathrm{X}$, assessment tool.

the ICU due to the severity of illness and hence cannot uniformly identify patients at risk of malnutrition $(37,55)$. Also, performing a nutrition-focused physical assessment (NFPA) in ICU patients might not be accurate, since they frequently are intubated, sedated, and volume overloaded (55).

To date, the only two validated methods for measuring the loss of lean tissue in critically ill patients with severe edema have been in vitro neutron activation analysis (IVNAA) (41) and assessing differences in muscle fiber area using repeated muscle biopsies (42). The former requires radiation and is not commonly available, and the latter is time-consuming and invasive. Both methods are only used in research settings.

Imaging techniques, such as $\mathrm{CT}$, magnetic resonance imaging (MRI) or ultrasound have recently been studied for muscle mass assessment $(43,44)$. Commonly performed on ICU patients, CT scans provide a more reliable measure of muscle mass in comparison with externally measured muscle circumferences (43), in these medically ill populations. The CT images can be combined with mathematical reconstruction algorithms to estimate the mass of individual muscle groups or the total-body skeletal muscle mass. Single-slice CT images in the L3 region can predict whole-body muscle and adipose tissue volume in healthy individuals and ICU populations (44). However, CT scans are not performed on every critically ill patient, as it is costly and involves radiation exposure for prospective evaluation of body composition (44). Ultrasonography is a new and promising non-volitional measure that enables identification of changes in muscle structure and morphology (56). It is noninvasive, inexpensive, and can be performed at the bedside. Studies have also shown good inter- and intra-observer reliability $(45,46)$. Campbell et al. (47) suggested that ultrasound could identify and possibly quantify muscle wasting in edematous patients with multiple organ failures. Other studies have also demonstrated that loss of muscle mass, determined by ultrasound, correlated negatively with the ICU length of stay $(29,57)$. Also, this measurement correlates well with CT scan evaluations. While the findings are promising, further studying of assessing muscle mass by using ultrasonography to predict mortality and poor outcomes of ICU patients is needed.

\section{Conclusions}

Low muscle mass is a strong predictor of poor outcomes in ICU patients. Studies have shown high mortality in ICU patients with low admission SCr levels. Although $\mathrm{SCr}$ levels can be used as a surrogate of muscle mass, it is influenced by other GFR- and non-GFR-related factors. Further studies are needed to implement insights of underlying mechanisms of an association between low $\mathrm{SCr}$ 
and mortality in the ICU patients as well as to evaluate if aggressive nutritional support in critically ill patients with low SCr levels can improve their mortality. Studies have demonstrated promising data on the uses of CT scans and ultrasonography for muscle mass measurement in ICU patients.

\section{Acknowledgements}

None.

\section{Footnote}

Provenance: This is an invited Perspective commissioned by the Section Editor Zhongheng Zhang (Department of Critical Care Medicine, Jinhua Municipal Central Hospital, Jinhua Hospital of Zhejiang University, Jinhua, China). Conflicts of interest: The authors have no conflicts of interest to declare.

\section{References}

1. Andrews R, Greenhaff P, Curtis S, et al. The effect of dietary creatine supplementation on skeletal muscle metabolism in congestive heart failure. Eur Heart J 1998;19:617-22.

2. Bagshaw SM, George C, Bellomo R, et al. A comparison of the RIFLE and AKIN criteria for acute kidney injury in critically ill patients. Nephrol Dial Transplant 2008;23:1569-74.

3. Ricci Z, Cruz D, Ronco C. The RIFLE criteria and mortality in acute kidney injury: A systematic review. Kidney Int 2008;73:538-46.

4. Thakar CV, Christianson A, Freyberg R, et al. Incidence and outcomes of acute kidney injury in intensive care units: a Veterans Administration study. Crit Care Med 2009;37:2552-8.

5. Thongprayoon C, Cheungpasitporn W, Harrison AM, et al. The comparison of the commonly used surrogates for baseline renal function in acute kidney injury diagnosis and staging. BMC Nephrol 2016;17:6.

6. Thongprayoon C, Cheungpasitporn W, Kittanamongkolchai W, et al. Optimum methodology for estimating baseline serum creatinine for the acute kidney injury classification. Nephrology (Carlton) 2015;20:881-6.

7. Thongprayoon C, Cheungpasitporn $\mathrm{W}$, Srivali N, et al. The impact of fluid balance on diagnosis, staging and prediction of mortality in critically ill patients with acute kidney injury. J Nephrol 2016;29:221-7.

8. Thongprayoon C, Cheungpasitporn W, Akhoundi A, et al. Actual versus ideal body weight for acute kidney injury diagnosis and classification in critically ill patients. BMC Nephrol 2014;15:176.

9. Schutte JE, Longhurst JC, Gaffney FA, et al. Total plasma creatinine: an accurate measure of total striated muscle mass. J Appl Physiol Respir Environ Exerc Physiol 1981;51:762-6.

10. Park J, Mehrotra R, Rhee CM, et al. Serum creatinine level, a surrogate of muscle mass, predicts mortality in peritoneal dialysis patients. Nephrol Dial Transplant 2013;28:2146-55.

11. Thomas ME, Blaine C, Dawnay A, et al. The definition of acute kidney injury and its use in practice. Kidney Int 2015;87:62-73.

12. Assy N, Kayal M, Mejirisky Y, et al. The changes in renal function after a single dose of intravenous furosemide in patients with compensated liver cirrhosis. BMC Gastroenterol 2006;6:39.

13. Lieu C, Anderson R. Serum creatinine: why lower may not be better. Crit Care Med 2007;35:2458-9.

14. De Waele JJ, Dumoulin A, Janssen A, et al. Epidemiology of augmented renal clearance in mixed ICU patients. Minerva Anestesiol 2015;81:1079-85.

15. Sime FB, Udy AA, Roberts JA. Augmented renal clearance in critically ill patients: etiology, definition and implications for beta-lactam dose optimization. Curr Opin Pharmacol 2015;24:1-6.

16. Cartin-Ceba R, Afessa B, Gajic O. Low baseline serum creatinine concentration predicts mortality in critically ill patients independent of body mass index. Crit Care Med 2007;35:2420-3.

17. Udy AA, Scheinkestel C, Pilcher D, et al. The Association Between Low Admission Peak Plasma Creatinine Concentration and In-Hospital Mortality in Patients Admitted to Intensive Care in Australia and New Zealand. Crit Care Med 2016;44:73-82.

18. Walther CP, Carter CW, Low CL, et al. Interdialytic creatinine change versus predialysis creatinine as indicators of nutritional status in maintenance hemodialysis. Nephrol Dial Transplant 2012;27:771-6.

19. Kalantar-Zadeh K, Streja E, Kovesdy CP, et al. The obesity paradox and mortality associated with surrogates of body size and muscle mass in patients receiving hemodialysis. Mayo Clin Proc 2010;85:991-1001.

20. Tonelli M, Klarenbach SW, Lloyd AM, et al. Higher estimated glomerular filtration rates may be associated 
with increased risk of adverse outcomes, especially with concomitant proteinuria. Kidney Int 2011;80:1306-14.

21. Carter CE, Gansevoort RT, Scheven L, et al. Influence of urine creatinine on the relationship between the albuminto-creatinine ratio and cardiovascular events. Clin J Am Soc Nephrol 2012;7:595-603.

22. Chronic Kidney Disease Prognosis Consortium, Matsushita K, van der Velde M, et al. Association of estimated glomerular filtration rate and albuminuria with all-cause and cardiovascular mortality in general population cohorts: a collaborative meta-analysis. Lancet 2010;375:2073-81.

23. Choi HM, Hyun YY, Lee KB, et al. High estimated glomerular filtration rate is associated with coronary artery calcification in middle-aged Korean men without chronic kidney disease. Nephrol Dial Transplant 2015;30:996-1001.

24. Shlipak MG, Sarnak MJ, Katz R, et al. Cystatin C and the risk of death and cardiovascular events among elderly persons. N Engl J Med 2005;352:2049-60.

25. Griffiths RD. Muscle mass, survival, and the elderly ICU patient. Nutrition 1996;12:456-8.

26. Lightfoot A, McArdle A, Griffiths RD. Muscle in defense. Crit Care Med. 2009;37:S384-90.

27. DeFronzo RA, Jacot E, Jequier E, et al. The effect of insulin on the disposal of intravenous glucose. Results from indirect calorimetry and hepatic and femoral venous catheterization. Diabetes 1981;30:1000-7.

28. Mogensen KM, Robinson MK, Casey JD, et al. Nutritional Status and Mortality in the Critically Ill. Crit Care Med 2015;43:2605-15.

29. Gruther W, Benesch T, Zorn C, et al. Muscle wasting in intensive care patients: ultrasound observation of the $M$. quadriceps femoris muscle layer. J Rehabil Med 2008;40:185-9.

30. Ix JH, de Boer IH, Wassel CL, et al. Urinary creatinine excretion rate and mortality in persons with coronary artery disease: the Heart and Soul Study. Circulation 2010;121:1295-303.

31. Heymsfield SB, McManus C, Stevens V, et al. Muscle mass: reliable indicator of protein-energy malnutrition severity and outcome. Am J Clin Nutr 1982;35:1192-9.

32. Englesbe MJ, Patel SP, He K, et al. Sarcopenia and mortality after liver transplantation. J Am Coll Surg 2010;211:271-8.

33. Park SW, Goodpaster BH, Lee JS, et al. Excessive loss of skeletal muscle mass in older adults with type 2 diabetes. Diabetes Care 2009;32:1993-7.

34. Gamrin L, Essén P, Forsberg AM, et al. A descriptive study of skeletal muscle metabolism in critically ill patients: free amino acids, energy-rich phosphates, protein, nucleic acids, fat, water, and electrolytes. Crit Care Med 1996;24:575-83.

35. Miller SC, Ito H, Blau HM, et al. Tumor necrosis factor inhibits human myogenesis in vitro. Mol Cell Biol 1988;8:2295-301.

36. Constantin D, McCullough J, Mahajan RP, et al. Novel events in the molecular regulation of muscle mass in critically ill patients. J Physiol 2011;589:3883-95.

37. Coltman A, Peterson S, Roehl K, et al. Use of 3 tools to assess nutrition risk in the intensive care unit. JPEN J Parenter Enteral Nutr 2015;39:28-33.

38. Pickkers P, de Keizer N, Dusseljee J, et al. Body mass index is associated with hospital mortality in critically ill patients: an observational cohort study. Crit Care Med 2013;41:1878-83.

39. Cerra FB, Benitez MR, Blackburn GL, et al. Applied nutrition in ICU patients. A consensus statement of the American College of Chest Physicians. Chest 1997;111:769-78.

40. Kuzuya M, Izawa S, Enoki $H$, et al. Is serum albumin a good marker for malnutrition in the physically impaired elderly? Clin Nutr 2007;26:84-90.

41. Hill GL. Implications of critical illness, injury, and sepsis on lean body mass and nutritional needs. Nutrition 1998;14:557-8.

42. Burnham EL, Moss M, Ziegler TR. Myopathies in critical illness: characterization and nutritional aspects. J Nutr 2005;135:1818S-1823S.

43. Ohkawa S, Odamaki M, Yoneyama T, et al. Standardized thigh muscle area measured by computed axial tomography as an alternate muscle mass index for nutritional assessment of hemodialysis patients. Am J Clin Nutr 2000;71:485-90.

44. Moisey LL, Mourtzakis M, Cotton BA, et al. Skeletal muscle predicts ventilator-free days, ICU-free days, and mortality in elderly ICU patients. Crit Care 2013;17:R206.

45. Tillquist M, Kutsogiannis DJ, Wischmeyer PE, et al. Bedside ultrasound is a practical and reliable measurement tool for assessing quadriceps muscle layer thickness. JPEN J Parenter Enteral Nutr 2014 Sep;38:886-90.

46. Baldwin CE, Paratz JD, Bersten AD. Diaphragm and peripheral muscle thickness on ultrasound: intrarater reliability and variability of a methodology using non-standard recumbent positions. Respirology 2011;16:1136-43.

47. Campbell IT, Watt T, Withers D, et al. Muscle thickness, 
measured with ultrasound, may be an indicator of lean tissue wasting in multiple organ failure in the presence of edema. Am J Clin Nutr 1995;62:533-9.

48. Kashani K, Kukralova L, Kashyap R, et al. A novel index for prediction of muscle mass and ICU outcomes. Am J Respir Crit Care Med 2015;191:A2284.

49. Simpson F, Doig GS; Early PN Trial Investigators Group. Physical assessment and anthropometric measures for use in clinical research conducted in critically ill patient populations: an analytic observational study. JPEN J Parenter Enteral Nutr 2015;39:313-21.

50. Braunschweig CA, Sheean PM, Peterson SJ, et al. Exploitation of diagnostic computed tomography scans to assess the impact of nutrition support on body composition changes in respiratory failure patients. JPEN J Parenter Enteral Nutr 2014;38:880-5.

51. Brewster DJ, Strauss BJ, Crozier TM. Measuring visceral fat, subcutaneous fat and skeletal muscle area changes by computed tomography in acute pancreatitis: a retrospective, single-centre study. Crit Care Resusc

Cite this article as: Thongprayoon C, Cheungpasitporn W, Kashani K. Serum creatinine level, a surrogate of muscle mass, predicts mortality in critically ill patients. J Thorac Dis 2016;8(5):E305-E311. doi: 10.21037/jtd.2016.03.62
2014;16:42-7.

52. Prado CM, Heymsfield SB. Lean tissue imaging: a new era for nutritional assessment and intervention. JPEN J Parenter Enteral Nutr 2014;38:940-53.

53. Reid CL, Campbell IT, Little RA. Muscle wasting and energy balance in critical illness. Clin Nutr 2004;23:273-80.

54. Manning EM, Shenkin A. Nutritional assessment in the critically ill. Crit Care Clin 1995;11:603-34.

55. Fischer M, JeVenn A, Hipskind P. Evaluation of muscle and fat loss as diagnostic criteria for malnutrition. Nutr Clin Pract 2015;30:239-48.

56. Parry SM, El-Ansary D, Cartwright MS, et al. Ultrasonography in the intensive care setting can be used to detect changes in the quality and quantity of muscle and is related to muscle strength and function. J Crit Care 2015;30:1151.e9-e14.

57. Puthucheary ZA, Rawal J, McPhail M, et al. Acute skeletal muscle wasting in critical illness. JAMA 2013;310:1591-600. 